\title{
Fatores associados às alterações da função pulmonar em trabalhadores de indústria de cerâmica
}

\author{
Factors associated with alterations in lung function \\ among workers in the ceramics industry
}

Viviane Aparecida Martins Mana Salicio ${ }^{1}$

Clovis Botelho ${ }^{2}$

Ageo Mário Cândido da Silva ${ }^{2}$

Marcos Adriano Salicio ${ }^{3}$

${ }^{1}$ Curso de Fisioterapia,

Universidade de Cuiabá. Av.

Beira Rio 3.100, Jardim

Europa. 78015-480

Cuiabá MT.

vivimana@hotmail.com

${ }^{2}$ Programa de Mestrado em

Saúde Coletiva, Instituto de

Saúde Coletiva,

Universidade Federal de

Mato Grosso.

${ }^{3}$ Curso de Fisioterapia,

Centro Universitário de

Várzea Grande.

\begin{abstract}
This article seeks to assess lung function in workers of ceramic industries in the municipality of Várzea Grande - Mato Grosso. A crosssectional study of 183 workers was conducted in the ceramics town of Várzea Grande (MT). A structured questionnaire was used to identify socio-demographic factors, environmental factors and smoking. and spirometry was performed to evaluatie the pulmonary function. Descriptive analysis and bivariate and multiple Poisson regression were performed. The workers are mostly male (94\%) with a predominance of individuals aged 39 years $(74.9 \%)$. The prevalence of respiratory symptoms was $44.9 \%, 14.8$ patients with symptoms considered serious and $30.1 \%$ with non-serious symptoms. Associations were found of abnormal pulmonary function with the variables of exposure time and substance inhaled. Employees with four or more years of exposure had 1.99 times more lung function alterations than individuals with exposure time of up to 3 years; individuals who had inhaled dust and mold release had 2.97 times higher alteration in lung function. The change in lung function in workers assessed is related to longer exposure and inhalation of dust and mold release.
\end{abstract}

Key words Occupational health, Spirometry, Ceramics
Resumo O objetivo deste artigo é analisar a função pulmonar em trabalhadores de indústrias de cerâmica no município de Várzea Grande, Mato Grosso. Estudo transversal realizado com 183 trabalhadores de indústrias de cerâmica desse município. Para identificação dos fatores sociodemográficos, fatores ambientais e do tabagismo foi utilizado um questionário estruturado e para avaliação da função pulmonar foi realizada a espirometria. Foram realizadas análises descritiva, bivariada e regressão múltipla de Poisson. Os trabalhadores em sua maioria são do sexo masculino (94\%) predominando indivíduos com idade até 39 anos (74,9\%). A prevalência de sintomáticos respiratórios foi de $44,9 \%$, sendo $14,8 \%$ portadores de sintomas considerados como graves e $30,1 \%$ sintomas não graves. Foram encontradas associações de função pulmonar alterada com as variáveis tempo de exposição e substância inalada. Os trabalhadores com 4 ou mais anos de exposição apresentaram 1,99 vezes mais função pulmonar alterada que os indivíduos com tempo de exposição de até 3 anos; os indivíduos que inalaram poeira e desmoldante apresentaram 2,97 vezes mais alteração da função pulmonar. Esta, nos trabalhadores avaliados, está relacionada ao maior tempo de exposição e à inalação de poeira e desmoldante.

Palavras-chave Saúde do trabalhador, Espirometria, Cerâmicas 


\section{Introdução}

O Estado de Mato Grosso possui 130 indústrias de cerâmica vermelha em atividade, das quais vinte e sete estão localizadas no polo cerâmico de Várzea Grande, empregando cerca de 1.000 trabalhadores. As estimativas do setor é que sua capacidade instalada dobre nos próximos dois anos devido às construções e obras derivadas das melhorias para a Copa do Mundo de 2014, já que a capital do Estado, Cuiabá, será uma das sedes do evento. A realidade das indústrias deste setor não é diferente da dos demais estados brasileiros, com baixo nível tecnológico, mão-deobra não qualificada e baixa escolaridade dos trabalhadores ${ }^{1}$.

As plantas industriais brasileiras de placas cerâmicas apresentam, atualmente, dois tipos de processos: moagem por via seca e moagem por via úmida. Cada tipo de processo expõe de forma diferenciada os trabalhadores ao risco de doenças respiratórias, em razão da diferente composição mineral das argilas utilizadas como matéria-prima (argilas branca e vermelha) e das diferentes fontes de geração de poeira ${ }^{2}$.

A indústria de cerâmica utiliza, na produção de tijolos e telhas, rotinas de trabalho que expõem seus trabalhadores a altíssimas temperaturas. Os produtos cerâmicos são queimados em fornos por períodos de até 36 horas, sendo que nesse processo a temperatura varia de $280^{\circ} \mathrm{C}$ à $890^{\circ} \mathrm{C}^{3}$. Período em que os trabalhadores inalam partículas de poeiras e fumaça que podem depositar-se nas vias aéreas e interferir nos mecanismos de defesa do pulmão ou depositar-se nas pequenas vias aéreas, contribuindo para o desenvolvimento de diversas doenças do aparelho respiratório ${ }^{4}$.

O desmoldante é um produto utilizado para untar as formas das máquinas que dão molde às telhas e tijolos. Na sua composição eram muito utilizados os compostos de óleos leves contendo ácido orgânico, que, mesmo com o aperfeiçoamento de sua composição química, continua contendo óleos vegetais ou minerais ${ }^{5}$. Estes, são constituídos por vaselinas pesadas e polímeros de resinas epóxi que podem conter solventes voláteis incluindo benzeno e outros hidrocarbonetos aromáticos policíclicos (PAH), todos com potenciais efeitos tóxicos sobre o organismo. $\mathrm{O}$ benzeno é um composto orgânico volátil que podem causar problemas respiratórios além de sonolência, tontura, fadiga até narcose e morte ${ }^{6}$. Os PAH, do inglês polycyclic Aromatic Hydrocarbons, constituem uma classe de compostos orgânicos caracterizada por substâncias que apresentam dois ou mais anéis aromáticos conjugados, gerados pela combustão incompleta de material orgânico e que apresentam grande potencial tóxico sobre a saúde humana ${ }^{7,8}$.

O desmoldante também libera inúmeras substâncias tóxicas no ambiente quando aquecido pelas chapas das máquinas. Um dos principais poluentes é o monóxido de carbono (CO) que pode acarretar lesões nas vias áreas superiores e inferiores, levando à dispnéia como seu principal sintoma respiratório9. A nocividade do CO no organismo deriva do fato deste gás possuir uma afinidade 250 vezes maior do que o oxigênio $\left(\mathrm{O}_{2}\right)$ com a hemoglobina ( $\mathrm{Hb}$ ), que é responsável pelo transporte de $\mathrm{O}_{2}$ aos tecidos. A ligação do $\mathrm{CO}$ à $\mathrm{Hb}$ forma o composto carboxiemoglobina $(\mathrm{COHb})$, impedindo a fixação do $\mathrm{O}_{2}$ e prejudicando o transporte do suprimento deste para as células do corpo ${ }^{10,11}$. O comprometimento da capacidade de transporte de $\mathrm{O}_{2}$ para os tecidos limita a capacidade funcional do organismo, restringindo o desempenho físico, situação em que sua demanda está aumentada ${ }^{10,12}$. Também a fumaça originada neste processo contém grandes concentrações de material particulado e outras substâncias irritantes das vias aéreas e olhos ${ }^{13}$.

A poluição ambiental aparece como uma característica das indústrias de cerâmicas uma vez que no processo de queima do produto é possível observar alto índice de fumaça, cinza e materiais particulados e irritantes primários das vias aéreas superiores e olhos, resultantes dos vários tipos de materiais utilizados na alimentação dos fornos. Para minimizar esse quadro, é indicada a utilização de filtros nas chaminés e máscaras com elementos filtrantes adequados. Deve existir sistema de ventilação e/ou exaustão adequado para evitar a concentração de contaminantes nas áreas de produção e estocagem. Assim, quanto aos riscos ambientais, a degradação do meio ambiente propiciada pela indústria cerâmica pode levar ao alto consumo de energia vegetal com esgotamento de áreas legalizadas para exploração. Além disso, o descarte dos resíduos com arraste de elementos poluentes tais como metais pesados ou substâncias tóxicas podem ser grandes poluidores do meio ambiente ${ }^{14}$

Alguns métodos podem ser utilizados para avaliação dos agravos respiratórios de trabalhadores expostos aos poluentes do ar respirado, sendo os mais utilizados os questionários de detecção de sintomas respiratórios e a espirometria que avalia a função pulmonar ${ }^{15}$. A espirometria é um teste que avalia as condições mecâ- 
nicas do pulmão e da caixa torácica com base em um traçado representativo dos volumes ou fluxos de ar inspirados ou expirados durante manobras respiratórias específicas, sendo bastante utilizada na prevenção, identificação e quantificação dos distúrbios respiratórios ${ }^{16}$.

Neste contexto, o objetivo do presente estudo foi avaliar a associação entre fatores ambientais e relacionados ao trabalho e alterações da função pulmonar dos trabalhadores de indústrias de cerâmica no município de Várzea Grande (MT).

\section{Metodologia}

Estudo transversal com 183 trabalhadores de indústrias de cerâmica do município de Várzea Grande (MT) no ano de 2010. A coleta de dados foi realizada por meio de aplicação de questionários que continham informações sociodemográficas, história de sintomas e doenças respiratórias, tabagismo, além de informações sobre as atividades laborais. Após a aplicação dos questionários, foram avaliadas as funções pulmonares dos participantes através do teste espirométrico. Os critérios de inclusão para a seleção dos participantes foram ter 18 anos ou mais, não apresentar patologias respiratórias graves, não estar em período gestacional (apenas para mulheres) e estar trabalhando em cerâmicas do município de Várzea Grande. Todos os trabalhadores avaliados concordaram em participar do estudo mediante assinatura do termo de consentimento.

A avaliação da função pulmonar foi obtida pela utilização do espirômetro portátil da Marca Ferraris, ano 2008, série 2008K3155, com calibração diária, sete litros de capacidade e registro gráfico direto em conformidade com critérios da American Thoracic Society (ATS ${ }^{17}$. O espirômetro permite traçar curvas de expiração forçada e de ciclos respiratórios basais, a partir das quais são determinados os valores dos parâmetros referentes à função do sistema respiratório ${ }^{18}$. A espirometria foi realizada por um profissional técnico treinado logo após a aplicação do questionário; os trabalhadores permaneceram na posição sentada, com tronco ereto e a cabeça em posição neutra e fixa, utili-zando-se um clipe nasal. Os mesmos foram solicitados a inspirar profundamente até a capacidade pulmonar total (CPT) e expirar o mais forte e rápido possível até o volume residual (VR). Todos os participantes receberam estímulo verbal para máximo esforço durante toda a manobra, sendo a expiração in- terrompida ao final de 6 segundos, após atingir um platô. Após os esclarecimentos devidos iniciaram-se os registros gráficos, seguindo as recomendações preconizadas pela $\mathrm{ATS}^{17}$. Para obtenção da curva ideal cada trabalhador realizou no mínimo três manobras de capacidade vital forçada (CVF). Esta e o volume expiratório forçado no primeiro segundo $\left(\mathrm{VEF}_{1}\right)$ aceitos foram os melhores valores de cada trabalhador respeitando os critérios de aceitabilidade segundo o consenso brasileiro de espirometria. O fluxo expiratório entre 25-75\% (FEF25-75) foi derivado da curva com melhor soma do $\mathrm{VEF}_{1}$ com a CVF. Também foram coletadas informações sobre peso e altura dos participantes.

A análise de dados foi realizada através dos programas SPSS versão 15.0 e STATA versão 11.0. Foram realizadas análise descritiva e bivariada que verificou as associações entre as variáveis independentes e a função pulmonar alterada dos trabalhadores. A presença de confundimento foi investigada através de modelos estratificados comparando-se razões de prevalências brutas com ajustadas, segundo a metodologia de MantelHaenszel. Utilizou-se o teste de Qui-quadrado para razões de prevalências com intervalo de $95 \%$ de confiança pelo método de Mantel-Haenszel (IC95\%), ou o Exato de Fisher quando indicado.

$\mathrm{Na}$ terceira fase foi construído um modelo de regressão múltipla de Poisson para controlar simultaneamente o efeito das diversas covariáveis para a explicação da função pulmonar alterada. Incluiu-se no modelo as variáveis que na análise bivariada apresentaram associações com p-valor igual ou inferior a $0,20^{19}$. As variáveis sexo, faixa etária e tabagismo foram cosideradas, a priori, como importantes para o ajustamento do modelo de regressão, tendo sido mantidas no modelo final independente de sua significância estatística. Foram consideradas como associações estatisticamente significantes aquelas cujo p-valor tenha sido menor ou igual a 0,05 .

Este estudo foi aprovado pela Comissão de Ética em Pesquisa do Hospital Universitário Júlio Muller (CEP/HUJM).

\section{Resultados}

Houve um predomínio de trabalhadores do sexo masculino, com idade de até 39 anos, de raça/cor não branca e casados. Também observou-se uma maior prevalência de indivíduos que estudaram 5 anos e mais e quase metade dos participantes tinha renda familiar per capita menor de $1 / 2$ sa- 
lário mínimo (Tabela 1). Um quarto dos trabalhadores era fumante regular. Além disso, quase metade referiu ter sintomas respiratórios e, destes, 1/3 apresentavam sintomas graves (dispnéia, chiado e ronqueira).

$\mathrm{Na}$ análise bivariada observou-se uma maior proporção de alteração da função pulmonar dos forneiros em relação às demais ocupações nas cerâmicas $[\mathrm{RP}=1,87(0,97-3,63)]$, porém a diferença foi estatisticamente limítrofe ( $\mathrm{p}=$ $0,067)$. Notou-se também que trabalhadores com maior tempo de exposição (4 anos ou mais), apresentaram prevalência de alteração da função pulmonar 99\% maior [RP = 1,99 $(1,05$ $3,77)]$. Quanto à jornada de trabalho, o grupo que trabalhava mais de oito horas diárias apresentou maior prevalência de função pulmonar alterada que o grupo que trabalhava por menos tempo, ainda que sem significância estatística [RP $=1,40(0,49-3,99)]$. Quando se analisou a substância inalada no ambiente de trabalho, os indivíduos que inalam poeira e desmoldante apresentaram mais episódios de alteração da função pulmonar $[\mathrm{RP}=2,97(1,09-8,10)]$, tendo sido

Tabela 1. Características dos trabalhadores de indústrias de cerâmica do município de Várzea Grande (MT), 2010.

\begin{tabular}{lrr}
\hline \multicolumn{1}{c}{ Variáveis } & N & $\%$ \\
\hline Sexo & & \\
$\quad$ Masculino & 172 & 94,0 \\
Feminino & 11 & 6,0 \\
$\quad$ Total & 183 & 100 \\
Faixa etária & 137 & 74,9 \\
$\quad$ Até 39 anos & 46 & 25,1 \\
40 anos e mais & 183 & 100 \\
Total & & \\
Raça & 56 & 30,6 \\
$\quad$ Branca & 127 & 69,4 \\
Não branca & 183 & 100 \\
Total & & \\
Estado Civil & 55 & 30,1 \\
$\quad$ Solteiro & 128 & 69,9 \\
Casado & 183 & 100 \\
$\quad$ Total & & \\
Anos de estudo & 51 & 27,9 \\
$\quad$ Até 4 anos & 132 & 72,1 \\
5 anos e mais & 183 & 100 \\
Total & & \\
Renda per capita & 88 & 48,1 \\
Até 1/2 salário mínimo & 95 & 51,9 \\
Mais de 1/2 salário mínimo & 183 & 100 \\
Total & & \\
\hline &
\end{tabular}

este resultado estatisticamente significante. Em relação ao tabagismo, indivíduos fumantes apresentaram uma maior prevalência de alteração da função pulmonar quando comparado aos exfumantes $[\mathrm{RP}=1,38(0,66-2,88)]$, porém esta diferença também não foi estatisticamente significativa. Também houve uma maior prevalência de função pulmonar alterada entre aqueles que relataram presença de doenças e sintomas respiratórios, sem diferença estatisticamente significante $[R P=1,19(0,18-7,50)]$ (Tabela 2$)$.

No modelo múltiplo final de Poisson, ajustado por sexo, idade e hábito tabágico, as variáveis tempo de exposição e referência à exposição, à poeira e ao desmoldante se mantiveram com associações estatisticamente significantes (Tabela 3 ).

\section{Discussão}

Identificou-se uma associação estatisticamente significante entre total de anos trabalhados nas cerâmicas e alteração da função pulmonar. Quanto maior o tempo de exposição aos poluentes do ar presentes no processo produtivo das cerâmicas, maior a prevalência de aparecimento de doença respiratória, conforme relatam Castro et al. ${ }^{20}$, Souza e Quelhas ${ }^{21}$. Já em relação à jornada de trabalho diária, não foi observada diferença na função pulmonar entre os grupos que trabalhavam até 8 horas e mais de 8 horas diárias. Estes resultados também são concordantes com o estudo de Costa et al. ${ }^{22}$ que relataram que a extensão da jornada de trabalho diária não interferiu na função pulmonar dos trabalhadores avaliados. Contudo, Castro et al. ${ }^{23}$, avaliaram a carga horária semanal juntamente com o número de anos trabalhados em cada emprego, obtendo associação entre alterações radiológicas e funcionais do pulmão e duração da jornada de trabalho.

Encontrou-se associação com estatística limítrofe entre a atividade de forneiro e alteração da função pulmonar de maneira semelhante à encontrada por Rondon et al. ${ }^{24}$, em pesquisa realizada em cerâmicas da mesma cidade deste presente estudo. Segundo Algranti et al. ${ }^{25}$, forneiros em indústria de cerâmicas podem ser expostos a hidrocarbonetos aromáticos policíclicos e asbesto, substâncias reconhecidamente tóxicas, podendo levar a doenças respiratórias, inclusive câncer de pulmão.

Em relação à inalação de poeira e desmoldante durante a atividade ocupacional, os trabalhadores que relataram essa exposição apresentaram maior proporção de alterações da função 
Tabela 2. Prevalência, razão de prevalência (RP) e intervalo de confiança (IC 95\%) de função pulmonar alterada segundo algumas características individuais e do ambiente de trabalho dos trabalhadores de indústrias de cerâmica do município de Várzea Grande (MT), 2010.

\begin{tabular}{|c|c|c|c|c|}
\hline Variáveis & $\mathrm{n} / \mathrm{N}$ & Prevalência & RP (IC 95\%) & p-valor \\
\hline \multicolumn{5}{|c|}{ Ocupações dos trabalhadores } \\
\hline Outros & $21 / 146$ & 14,4 & 1,00 & \\
\hline Forneiro & $10 / 37$ & 27,0 & $1,87(0,97-3,63)$ & 0,067 \\
\hline \multicolumn{5}{|l|}{ Tempo de exposição } \\
\hline Até 3 anos & $19 / 139$ & 13,7 & 1,00 & \\
\hline 4 anos ou mais & $12 / 44$ & 27,3 & $1,99(1,05-3,77)$ & 0,036 \\
\hline \multicolumn{5}{|l|}{ Tabagismo } \\
\hline Não fumante & $14 / 93$ & 15,1 & 1,00 & \\
\hline Ex-fumante & $7 / 42$ & 16,7 & $1,10(0,48-2,54)$ & 0,811 \\
\hline Fumante & $10 / 48$ & 20,8 & $1,38 \quad(0,66-2,88)$ & 0,388 \\
\hline \multicolumn{5}{|l|}{ Jornada de trabalho diário } \\
\hline Até 8 horas & $28 / 170$ & 16,5 & 1,00 & \\
\hline Mais de 8 horas & $3 / 13$ & 23,1 & $1,40(0,49-3,99)$ & $0,382^{*}$ \\
\hline \multicolumn{5}{|l|}{ Substâncias inaladas } \\
\hline Outros & $4 / 56$ & 7,1 & 1,00 & \\
\hline Poeira e desmoldante & $27 / 127$ & 21,3 & $2,97(1,09-8,10)$ & $0,012^{*}$ \\
\hline \multicolumn{5}{|l|}{ Doenças respiratórias } \\
\hline Não & $1 / 7$ & 14,3 & 1,00 & \\
\hline $\operatorname{Sim}$ & $30 / 176$ & 17,0 & $1,19(0,18-7,50)$ & $0,662^{\star}$ \\
\hline \multicolumn{5}{|l|}{ Sintomas respiratórios } \\
\hline Não & $17 / 101$ & 16,8 & 1,00 & \\
\hline Sintomas não graves & $9 / 55$ & 16,4 & $0,97(0,46-2,03)$ & 0,940 \\
\hline Sintomas graves & $5 / 27$ & 18,5 & $1,12(0,45-2,71)$ & $0,517^{*}$ \\
\hline
\end{tabular}

${ }^{*}$ Teste exato de Fisher

pulmonar do que os demais. Estes resultados também foram encontrados em estudos que demonstraram que a indústria de cerâmica expõe seus trabalhadores a grandes quantidades de material particulado presente na poeira e nos produtos usados nas cerâmicas, o que leva, consequentemente, à maior ocorrência de doenças respiratórias do que a população em geral ${ }^{25,26}$. Além disso, a expansão recente e crescente dessa indústria na região pode sugerir que estejam coexistindo métodos primitivos que mesclam fumaça de queima de madeira, fumos de óleos combustíveis e fumaça associada à poeira de desmoldantes. O processo de queima de madeira se dá em vários estágios, liberando diversos compostos de oxidação incompleta e formando várias partículas orgânicas. Esta exposição múltipla à fumaça de queima de madeira e de hidrocarbonetos dos óleos combustíveis dos fornos, adicionada aos vapores dos desmoldantes, pode gerar incompatibilidades idiossincráticas levando ao aumento da ocorrência de reações adversas desconhecidas e agravos paradoxais na saúde dos trabalhadores. Outra possível explicação para
Tabela 3. Modelo final de regressão de Poisson para função pulmonar alterada em trabalhadores de indústrias de cerâmica do município de Várzea Grande (MT), 2010.

\begin{tabular}{cccc}
\hline Variáveis & RP & IC 95\% & p valor \\
\hline $\begin{array}{c}\text { Tempo de exposição } \\
4 \text { anos ou mais/até } 3 \text { anos }\end{array}$ & 1,84 & $(1,00-3,39)$ & 0,049 \\
$\begin{array}{c}\text { Substâncias inaladas } \\
\text { Poeira e desmoldante/outros }\end{array}$ & 3,07 & $(1,00-9,41)$ & 0,050 \\
\hline
\end{tabular}

Ajustado por sexo, idade e hábito tabágico.

essa exposição ocupacional é que, apesar dos trabalhadores terem relatado uso de Equipamento de Proteção Individual (EPI), como máscaras e luvas, não foi observada a sua utilização pela maioria dos funcionários durante o nosso trabalho de campo, apesar de que, sob o ponto de vista de conforto ergonômico, a sua utilização se torna quase inviável sob as extremas temperaturas próximas aos fornos. Além disso, alguns autores sugerem a existência de grande variação na capacidade de retenção de poluentes dos EPI, in- 
dicando que mesmo o uso constante de máscaras pode não promover o efeito protetor desejado desses equipamentos ${ }^{27}$. Também se percebeu a inexistência de sistemas de exaustão ou outros equipamentos de proteção coletivo que permitiriam a dispersão da neblina formada pelo desmoldante e demais poluentes e, consequentemente, o impedimento de sua inalação pelos funcionários que lidam diretamente com esses produtos.

Muitos profissionais da área de saúde desconhecem ou subestimam a etiologia ocupacional das doenças respiratórias, levando à subnotificação destes agravos pelos serviços de saúde. Além disso, se faz necessária a identificação do nexo causal entre a ocupação do trabalhador exposto e a ocorrência de doenças respiratórias para a viabilização de estratégias que favoreçam a prevenção e o prognóstico dessas doenças. Para tanto, são necessárias modificações do ambiente de trabalho para a diminuição do impacto na saúde dos trabalhadores sob risco ${ }^{28,29}$.

Apesar da identificação de função pulmonar alterada, percebeu-se uma pouca morbidade referida de doenças respiratórias pelos ceramistas. Esse fato pode ser explicado devido à grande rotatividade de funcionários e à maior proporção de indivíduos jovens nestas indústrias. Pode, neste caso, ter ocorrido o "viés do trabalhador saudável", quando existe uma seleção de indivíduos mais saudáveis para trabalhar nas empresas ${ }^{30}$. Contudo, Rondon et al. ${ }^{24}$ observaram uma maior prevalência de sintomáticos respiratórios e sintomáticos respiratórios graves, em estudo realizado na mesma base populacional.

Quanto à avaliação da função pulmonar, a literatura não é de toda concordante. Florêncio et al. ${ }^{31}$ avaliaram a função cardiorrespiratória de ceramistas com diagnóstico de silicose pulmonar, encontrando provas funcionais respiratórias alteradas em uma menor parte dos trabalhadores. Já Cowie et al. ${ }^{32}$ avaliaram mineiros expostos à sílica, encontrando perda da capacidade respiratória em toda a população estudada, da mesma maneira que o encontrado por Pinto ${ }^{33}$, ao analisar distúrbios ventilatórios de trabalhadores, onde os portadores de silicose apresentaram redução significativa nos índices espirométricos em relação àqueles com silicose simples. No entanto, Lopes et al. ${ }^{34}$, analisando trabalhadores expostos à sílica, encontrou a maioria deles com distúrbios respiratórios obstrutivos. Estes resultados podem ser explicados pela diferença da população avaliada e tipo de serviço médico (ambulatório ou inquéritos no local de trabalho, entre outros), onde, aqueles que procuram os serviços médicos apresentam maior carga de morbidade do que o trabalhador da cerâmica, em sua maioria, trabalhadores saudáveis.

Este estudo trouxe à luz o problema do processo saúde-doença na indústria ceramista em Mato Grosso. Todos os trabalhadores sintomáticos devem ser acompanhados e, seus sintomas, melhor diagnosticados, já que estes podem ser precursores de outros agravos respiratórios das vias aéreas inferiores. As exposições às altas temperaturas e poluentes podem ser reduzidas ou eliminadas com ações mais efetivas de vigilância em saúde do trabalhador no controle de poeiras e desmoldante nos ambientes de trabalho. O treinamento e a capacitação em saúde para o diagnóstico precoce dos sintomáticos respiratórios, bem como campanhas de educação em saúde para o uso obrigatório de EPI é de fundamental importância para a diminuição da morbidade por doenças respiratórias dos trabalhadores deste setor produtivo.

\section{Colaboradores}

VAMM Salicio e C Botelho participaram de todas as etapas da pesquisa; AMC Silva na análise estatística, revisão e estruturação do artigo; e, MA Salicio na concepção e na redação final do artigo. 


\section{Agradecimentos}

Agradecemos a todas as indústrias de cerâmicas que concordaram em participar desta pesquisa, bem como a todos os trabalhadores participantes. Aos professores Dr. Carlos Minayo Gomez e Dr. Hermano Albuquerque de Castro pelas contribuições importantes para o enriquecimento deste trabalho.

\section{Referências}

1. Indústria de Cerâmica cresce $10 \%$ ao ano, diz Anicer. Jornal Folha do Estado 2011 Ago 01; p. 1.

2. Associação Nacional dos Fabricantes de Cerâmica para Revestimento (ANFACER). Produção Brasileira de revestimento de cerâmicos e vendas de revestimentos Cerâmicos no Mercado Interno. 2011. [acessado 2012 mar 29]. Disponível em: http:/ /www.anfacer.org.br/principal.aspx?tela $=$ ucTela Conteudos\&idMenu=165

3. Gonçalves JP. Utilização do resíduo da indústria cerâmica para produção de concretos. Revista da Escola de Minas 2007; 60(4):639-644.

4. Bagatin E, Jardim JRB, Stirbulov R. Doença pulmonar obstrutiva crônica ocupacional. Jornal Bras. Pneumologia 2006; 32(2):35-40.

5. Maerschel R. Quality Needs for Concrete Mould Release. Berryessa Company, 2009. [acessado $2010 \mathrm{dez}$ 7]. Disponível em: http://www.berryessa.com.au/ uploads/QualityNeedsForConcreteMouldRelease. pdf

6. Phillips M, Gleeson K, Hughes J, Greenberg J, Cataneo R, Baker L, McVay WP. Volatile organic compounds in breath as markers of lung cancer: a crosssectional study. Lancet 1999; 353(9168):1930-1933.

7. Chun-Teh Li, Hsiao-Hsuan Mi, Wen-Jhy Lee, WenChun You,Ya-Fen Wang. PAH emission from the industrial boilers. J Hazard Mater 1999; 69(1):1-11.

8. Kuan-Foo Chang, Guor-Cheng Fang, Jhy-Cherng Chen, Yuh-Shen Wu. Atmospheric polycyclic aromatic hydrocarbons (PAHs) in Asia: a review from 1999 to 2004. Environ Pollut 2006; 142(3):388-396.

9. Gioda A, Aquino NFRA. Considerações sobre os estudos ambientais industriais e não industriais no Brasil: uma abordagem comparativa. Cad Saude Publica 2003; 19(5):1389-1397.

10. Fierro MA, O’Rourk MK, Burgess JL. Adverse health effects of exposure to ambient carbon monoxide. [atualizado 2001 set]. Mindfully. [acessado 2005 jun 20]. Disponível em: http://www.mindfully.org/ Health/Carbon-Monoxide-Health1sep01.htm

11. Foss ML, Keteyian SJ. Bases fisiológicas do exercício e do esporte. 6a Edição. Rio de Janeiro: Guanabara Koogan; 2000.

12. Dubbert P. Physical activity and exercise: recent advances and current challenges. J Consul Clin Pisych 2002; 70(3):526-536.

13. Ponsoni K, Mingireanov TR, Raddi MSG. Dispersão de bioaerossóis por aeradores em uma estação de tratamento de esgoto sanitário. Rev Ciênc Farm 2004; 25(2):115-118.

14. Cardoso M. As pequenas indústrias de cerâmica vermelha, ainda chamadas de "olarias", mantêm processos de trabalho arcaicos e desconhecem a cultura de segurança. Revista Proteção 2009:40-53.

15. American Thoracic Society (ATS). Standardization of spirometry. Am J Respir Crit Care Med 1995; 152: 77-121.

16. Pereira CAC. Espirometria. Jornal Bras. Pneumologia 2002; 28(3):1-238.

17. American Thoracic Society (ATS). Lung function testing: selection of reference values and interpretation. Am Rev Respir Dis 1991; 144:1202-1218. 
18. Pereira CA, Sato T, Rodrigues SC. New reference values for forced spirometry in white adults in Brazil. Jornal Bras. Pneumologia 2007; 33(4):397-406.

19. Kleinbaum DG, Kupper LL, Muller KE. Applied Regression Analysis and Other Multivariable Methods. $2^{\text {nd }}$ Edition. Belmont: Duxbury Press; 1988.

20. Castro HA, Souza CRM, Saraiva LG, Lemle A. Silicose: Correlação radiológica e funcional. Jornal Bras. Pneumologia 1992; 18(2):103.

21. Souza V F, Quelhas OLG. Avaliação e controle da exposição ocupacional à poeira na indústria da construção. Cien Saude Colet 2003; 8(3):801-808.

22. Costa M, Teixeira PJ, Freitas PF. Respiratory manifestations and respiratory diseases: prevalence and risk factors among pig farmers in Braço do Norte, Brazil. Jornal Bras. Pneumologia 2007; 33(4):380-388.

23. Castro HA, Vicentin G, Ribeiro PC, Mendonça ICT. Perfil respiratório de 457 trabalhadores expostos à poeira de sílica livre no Estado do Rio de Janeiro. Pulmão 2004; 13(2):81-85.

24. Rondon EN, Silva R MVG, Botelho C. Sintomas respiratórios como indicadores de estado de saúde em trabalhadores de indústrias de cerâmicas. Jornal Bras. Pneumologia 2011; 37(1):1-10.

25. Algranti E, Buschinelli JTP, Capitani EM. Câncer de pulmão Ocupacional. Jornal Bras. Pneumologia 2010; 36(6):784-794.

26. Lima MMTM, Camarini G. Silicose em trabalhadores do setor cerâmico: avaliação da poeira em processos de fabricação de revestimentos cerâmicos. Brasília: Fundacentro: Programa de prevenção de Silicose; 2003. p. 28-38.

27. Bagatin E, Neder JA. Doenças respiratórias ambientais e ocupacionais. Jornal Bras. Pneumologia 2006; 32(2):119-134.
28. Ponsoni K, Mingireanov TR, Raddi MSG. Eficiência de máscaras cirúrgicas como equipamento de proteção respiratória contra aerossóis bacterianos. Rev. Ciênc. Farm. Básica Apl. 2005; 26(2):157-158.

29. Kuschner WG, Stark P. Occupational lung disease, part 2. Postgrad Med 2003; 113(4):81-88.

30. Werneck GL, Almeida LM. Validade em estudos epidemiológicos. In: Medronho RA, Carvalho DM, Bloch KV, Luiz RR, Werneck GL, organizadores. Epidemiologia. São Paulo: Atheneu; 2004. p. 33-55.

31. Florêncio RT, Nery LE, Campos LB, Bagatin E, Jar$\operatorname{dim}$ JRB, Santos ML. Testes de exercício na avaliação funcional de ceramistas com silicose pulmonar. Rev. Bras. Saúde Ocup. 1989; 17(65):33-42.

32. Cowie RL. The influence of silicosis on deteriorating lung function in gold miners. Chest 1998; 113(2):340-343.

33. Pinto ALA. Avaliação espirométrica dos trabalhadores portadores de silicose atendidos no Hospital Universitário Antônio Pedro [tese]. Niterói: UFF; 2001.

34. Lopes AJ, Mogami R, Capone D, Tessarollo B, Melo PL, Jansen JM. Tomografia computadorizada de alta resolução na silicose: correlação com radiografia e testes de função pulmonar. Jornal Bras. Pneumologia 2008; 34(5):264-272.

Artigo apresentado em 25/02/2012

Aprovado em 30/03/2012

Versão final apresentada em 20/04/2012 